\title{
Reaction of Arylnitroso Derivatives: Synthesis of Arylimino 2,5-Dihydrofuran and Arylamino Fulvenes Derivatives
}

\author{
Stefania Fusi*, Donato Donati, Fabio Ponticelli \\ Dipartimento di Biotecnologie, Chimica e Farmacia, Università di Siena, Siena, Italy \\ Email: "stefania.fusi@unisi.it
}

Received 16 April 2016; accepted 25 June 2016; published 28 June 2016

Copyright @ 2016 by authors and Scientific Research Publishing Inc.

This work is licensed under the Creative Commons Attribution International License (CC BY). http://creativecommons.org/licenses/by/4.0/

c) (†) Open Access

\begin{abstract}
Herein we report the reactivity of $\mathrm{N}$-(2,4-dicyano-1,5-dimethyl-3-arylcyclopenta-2,4-dienyl)-2,2,2trifluoroacetamides and $\mathrm{N}, \mathrm{N}$-dimethyl-4-nitrosoaniline which provide compounds derived from Ehrlich-Sachs condensation, dihydrofuran derivatives and fulvene derivatives by adjusting the reaction conditions.
\end{abstract}

Keywords

Cyclopentadienes, Nitrsoarenes, Dihydrofuran, Fulvenes

\section{Introduction}

Densely functionalized cyclopentadienes $\mathbf{1}$ were recently prepared [1] and exploited as useful building blocks in synthetic chemistry. We have previously reported, in a preliminary account, the preparation of notable compounds using $\mathbf{1}$ as starter material. These syntheses involved the cycloaddition of $\mathbf{1}$ with a suitable reagent, followed by rearrangement (Figure 1) [2]. As an expansion of this study, we focused on arylnitroso derivatives as potential reagents for cyclopentadiene $\mathbf{1}$. Arylnitroso species were shown to give numerous reactions with unsaturated molecules, including cycloadditions and Aldol Michael [3]-[9]. In this context, the formation of 1,2oxazines would be of wide importance, due to the possibility of further chemical elaboration of this structure to access important scaffolds [10]. Herein we report our studies on the reactivity of N-(2,4-dicyano-1,5-dimethyl3-phenylcyclopenta-2,4-dienyl)-2,2,2-trifluoroacetamide and p- $\mathrm{NO}_{2}, 2,4-\mathrm{di}-\mathrm{NO}_{2}$, p-OMe phenyl derivatives in presence of nitrosobenzene or $N, N$-dimethyl-4-nitrosoaniline. This reaction is showed to give different products in dependence of the experimental conditions applied.

${ }^{*}$ Corresponding author.

How to cite this paper: Fusi, S., Donati, D. and Ponticelli, F. (2016) Reaction of Arylnitroso Derivatives: Synthesis of Arylimino 2,5-Dihydrofuran and Arylamino Fulvenes Derivatives. International Journal of Organic Chemistry, 6, 147-155.

http://dx.doi.org/10.4236/ijoc.2016.62016 


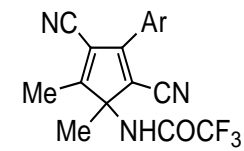

1

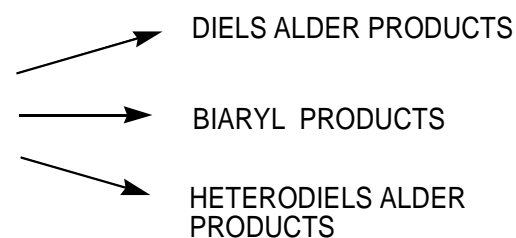

PRODUCTS

Figure 1. Synthetic target from cyclopentadiene $\mathbf{1}$.

\section{Experimental}

\subsection{Chemicals and Instruments}

General: Melting point was measured with a Kofler apparatus and was uncorrected. The ${ }^{1} \mathrm{H}$ and ${ }^{13} \mathrm{C}$ NMR spectra were recorded at $400.13 \mathrm{MHz}$ and $100.13 \mathrm{MHz}$ on a Bruker Advance DPX400. Chemical shifts are reported relative to tetramethylsilane at $0.00 \mathrm{ppm}$. ESI-MS spectra were recorded with a LCQ-DECA Thermo Finnigan instrument. TLC was performed on precoated $4 \times 6.7$ silica gel $60 \mathrm{~F} 254$ plates silica gel on aluminum (Aldrich) with detection by UV light. Column chromatography was carried out on Silica gel (E. Merck, $0.040-0.063 \mathrm{~mm}$ ). Microwave irradiations were conducted using a CEM Discover Synthesis Unit. Elemental analyses were performed on a Perkin Elmer PE 2004 Elemental Analyzer.

\subsection{General Method for the Preparation of Compounds 3a-d}

A mixture of compounds 1a-d $(0.1 \mathrm{mmol})$ and N,N-dimethyl-4-nitrosoaniline $2(0.12 \mathrm{mmol})$ in $\mathrm{CH}_{2} \mathrm{Cl}_{2}(3 \mathrm{ml})$ was stirred at room temperature for a week or in Sovirel tube in bath oil at $60^{\circ} \mathrm{C}$ for $7 \mathrm{~h}$. Alternatively the same mixture was heated under MW irradiation, $(150 \mathrm{~W})$ for 90 minutes. The crude reaction was purified by column chromatography (ethyl acetate/petroleum ether 1/4).

2(5Z)-5-((4-dimethylamino) phenylimino)-2acetyl-2-dihydro-2-methyl-4-phenylfuran-3-carbonitrile (3a)

Red oil (40\% yield) ${ }^{1} \mathrm{H}-\mathrm{NMR}\left(\mathrm{CDCl}_{3}\right): \delta$ (ppm) 1.80 (s, 3H, Me), 2.29 (s, 3H, Me), 2.98 (s, 6H, $\mathrm{NMe}_{2}$ ), 6.70 (d, $2 \mathrm{H}, \mathrm{J}=8.8 \mathrm{~Hz}, \mathrm{Ph}), 7.47-7.53(\mathrm{~m}, 5 \mathrm{H}, \mathrm{Ph}), 8.00-8.07$ (m, $2 \mathrm{H}, \mathrm{Ph}) .{ }^{13} \mathrm{C}-\mathrm{NMR}\left(\mathrm{CDCl}_{3}\right): \delta(\mathrm{ppm}) 21.52$, 24.67, 40.43, 92.33, 110.15, 112.03, 113.18, 118.44, 127.16, 129.38, 129.46, 131.29, 133.40, 147.08, 149.05, 152.49, 203.25. MS (ESI): $360\left(\mathrm{M}+\mathrm{H}^{+}\right)$; Anal. Calcd for $\mathrm{C}_{22} \mathrm{H}_{21} \mathrm{~N}_{3} \mathrm{O}_{2}$ : C, 73.52; H, 5.89; N, 11.69; Found: C, 73.28; H, 5.90; N, 11.73.

2'(5Z)-5-((4-dimethylamino) phenylimino)-2-acetyl-2,5-dihydro-2-methyl-4-(4'-nitrophenyl)furan-3-carbo nitrile (3b)

Crystalline red solid (35\% yield) (m.p. $146^{\circ} \mathrm{C}-147^{\circ} \mathrm{C}$ ) ${ }^{1} \mathrm{H}-\mathrm{NMR}\left(\mathrm{CDCl}_{3}\right): \delta$ (ppm) 1.83 (s, 3H, Me), 2.33 (s, 3H, Me), 2.99 (s, 6H, $\mathrm{NMe}_{2}$ ), 6.69 (d, 2H, J = 9.0 Hz, Ph), 7.51 (d, 2H, J = 9.0 Hz, Ph), 8.20 (d, 2H, J = $8.8 \mathrm{~Hz}$, $\mathrm{Ph}$ ), 8.32 (d, 2H, J = 8.8 Hz, Ph). ${ }^{13} \mathrm{C}-\mathrm{NMR}\left(\mathrm{CDCl}_{3}\right): \delta$ (ppm) 21.81, 24.90, 40.26, 92.82, 110.73, 111.04, 111.94,

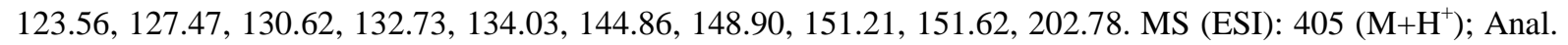
Calcd for $\mathrm{C}_{22} \mathrm{H}_{20} \mathrm{~N}_{4} \mathrm{O}_{4}$ : C, 63.34; H, 4.98; N, 13.85; Found: C, 63.55; H, 4.96; N, 13.89 .

2'(5Z)-5-((4-dimethylamino)phenylimino)-2-acetyl-2,5-dihydro-2-methyl-4-(2'-4'-nitrophenyl)furan-3-carbo nitrile (3c)

Blu solid (20\% yield) (m.p. $\left.149^{\circ} \mathrm{C}-151^{\circ} \mathrm{C}\right){ }^{1} \mathrm{H}-\mathrm{NMR}\left(\mathrm{CDCl}_{3}\right): \delta$ (ppm) 1.97 (s, 3H, Me), 2.15 (s, 3H, Me), 3.08 (s, 6H, NMe $), 6.70$ (d, 2H, J = 8.8 Hz, Ph), 7.36 (d, 2H, J = $8.8 \mathrm{~Hz}, \mathrm{Ph}$ ), 7.96 (d, 1H, J = 8.4 Hz, Ph), 8.67 $\left(\mathrm{d}, 1 \mathrm{H}, \mathrm{J}_{\mathrm{o}}=8.4 \mathrm{~Hz} \mathrm{~J}=1.6 \mathrm{~Hz}, \mathrm{Ph}\right), 9.20$ (bs, $\left.1 \mathrm{H}\right)$. MS (ESI): $450\left(\mathrm{M}+\mathrm{H}^{+}\right.$); Anal. Calcd for $\mathrm{C}_{22} \mathrm{H}_{20} \mathrm{~N}_{5} \mathrm{O}_{6}: \mathrm{C}$, 58.80; H, 4.26; N, 15.58; Found: C, 59.00; H, 4.24; N, 21.43.

2'(5Z)-5-((4-dimethylaminophenylimino)-2-acetyl-2,5-dihydro-2-methyl-4-(4'-methoxyphenyl)furan-3-carbon itrile (3d)

Crystalline orange-red solid (50\% yield) (m.p. $\left.148^{\circ} \mathrm{C}-150^{\circ} \mathrm{C}\right){ }^{1} \mathrm{H}-\mathrm{NMR}\left(\mathrm{CDCl}_{3}\right): \delta(\mathrm{ppm}) 1.77$ (s, 3H, Me), 2.26 (s, 3H, Me), 2.98 (s, 6H, $\mathrm{NMe}_{2}$ ), 3.86 (s, 3H, OMe), 6.69 (d, 2H, J = 8.8 Hz, Ph), 6.99 (d, 2H, J = 8.8 Hz, $\mathrm{Ph}), 7.49$ (d, 2H, J = $8.8 \mathrm{~Hz}, \mathrm{Ph}), 8.13(\mathrm{~d}, 2 \mathrm{H}, \mathrm{J}=8.8 \mathrm{~Hz}, \mathrm{Ph}) .{ }^{13} \mathrm{C}-\mathrm{NMR}\left(\mathrm{CDCl}_{3}\right): \delta(\mathrm{ppm}) 21.48,24.58,40.50$, 55.45, 92.14, 112.15, 113.14, 114.08, 116.07, 120.64, 127.05, 131.33, 133.67, 146.13, 149.04, 153.01, 162.13, 203.34. MS (ESI): $390\left(\mathrm{M}+\mathrm{H}^{+}\right)$; Anal. Calcd for $\mathrm{C}_{23} \mathrm{H}_{23} \mathrm{~N}_{3} \mathrm{O}_{3}$ : C, 70.93; $\mathrm{H}, 5.95 ; \mathrm{N}, 10.79$; Found: $\mathrm{C}, 71.16$; $\mathrm{H}$, $5.93 ; \mathrm{N}, 10.82$. 


\subsection{General Method for the Preparation of Compounds 4a-d, 5a-d, 6a-d}

Compounds 1a-d (0.1 mmol) and N,N-dimethyl-4-nitroso aniline $2(0.12 \mathrm{mmol})$ were heated under MW irradiation at $150^{\circ} \mathrm{C}$ for 15 minutes, $150 \mathrm{~W}$ solvent free or were heated in Sovirel tube in oil bath at $110^{\circ} \mathrm{C}$, solvent free for $3 \mathrm{~h}$. The crude reaction was purified by column chromatography (ethyl acetate/petroleum ether 1/4).

(5Z)-5-((4-(dimethylamino)phenylamino)methylene)-4-methyl-2-phenylcyclopenta-1,3-diene-1,3-dicarbonitril $e(\mathbf{4 a})$

Crystalline red solid (25\% yield) m.p. $255^{\circ} \mathrm{C}-258^{\circ} \mathrm{C}^{1} \mathrm{H}-\mathrm{NMR}\left(\mathrm{CDCl}_{3}\right): \delta(\mathrm{ppm}) 2.46$ (s, 3H, Me), 2.99 (s, 6H, $\mathrm{NMe}_{2}$ ), 6.73 (d, 2H, J = 8.6 Hz, Ph), 7.13 (d, 2H, J = 8.6 Hz, Ph), 7.38 (d, 1H, J = 7.5 Hz, Ph), 7.45 (t, 2H, J = $7.5 \mathrm{~Hz}, \mathrm{Ph}), 7.76$ (d, 2H, J = 7.5 Hz, Ph), 7.89 (d, 1H, J = 15.0 Hz), 9.30 (d, 1H, J = 15.0 Hz, NH). ${ }^{13} \mathrm{C}-\mathrm{NMR}$ $\left(\mathrm{CDCl}_{3}\right): \delta$ (ppm) 11.85, 40.46, 101.51, 112.89, 115.89, 117.22, 119.44, 120.91, 126.96, 127.82, 128.86, 132.50, 142.61, 146.51, 147.34, 149.54. MS (ESI): $353\left(\mathrm{M}+\mathrm{H}^{+}\right)$; Anal. Calcd for $\mathrm{C}_{23} \mathrm{H}_{20} \mathrm{~N}_{4}$ : C, 78.38; $\mathrm{H}, 5.72 ; \mathrm{N}, 15.90$; Found: C, 78.63; H, 5.70; N, 15.86 .

(Z)-N-((2,4-dicyano-5-methyl-3-phenyl-5-(2,2,2-trifluoroacetamido)cyclopenta-1,3-dien-1-yl)methylene)-4-(d imethylamino)aniline oxide (5a)

Red-violet solid (20\% yield) m.p. $205^{\circ} \mathrm{C}-208^{\circ} \mathrm{C}{ }^{1} \mathrm{H}-\mathrm{NMR}\left(\mathrm{CDCl}_{3}\right): \delta$ (ppm) 2.1 (s, 3H, Me), 3.07 (s, $6 \mathrm{H}$, $\mathrm{NMe}_{2}$ ), 6.65 (d, 2H, J = 9.4 Hz, Ph), 7.64 (d, 2H, J = 9.4 Hz, Ph), 7.48 - 7.53 (m, 3H, Ph), 7.72 - 7.75 (m, 2H, $\mathrm{Ph}), 8.11(\mathrm{~s}, 1 \mathrm{H}), 8.20(\mathrm{~s}, 1 \mathrm{H}, \mathrm{NH})$. MS-ESI: $480\left(\mathrm{M}+\mathrm{H}^{+}\right), 502\left(\mathrm{M}+\mathrm{Na}^{+}\right)$; Anal. Calcd for $\mathrm{C}_{25} \mathrm{H}_{20} \mathrm{~F}_{3} \mathrm{~N}_{5} \mathrm{O}_{2}$ : C, 62.63; H, 4.20; N, 14.61. Found: C,62.60; H,4.22; N,14.63.

$\mathrm{N}$-(2-((E)-(4-(dimethylaminophenylimino)methyl)-3,5-dicyano-1-methyl-4-phenylcyclopenta-2,4-dienyl)-2,2,2 -trifluoroacetamide (6a)

Blu-violet solid (22\% yield) m.p. $215^{\circ} \mathrm{C}-218^{\circ} \mathrm{C}{ }^{1} \mathrm{H}-\mathrm{NMR}\left(\mathrm{CDCl}_{3}\right): \delta$ (ppm) 1.91 (s, 3H, Me), 3.06 (s, 6H, $\mathrm{NMe}_{2}$ ), 6.69 (d, 2H, J = 9.0 Hz, Ph), 7.06 (s, 1H, NH), 7.35 (d, 2H, J = 9.0 Hz, Ph), 7.48 - 7.54 (m, 3H, Ph), 7.76 - 8.01 (m, 2H, Ph), 8.65 (s, 1H). MS-ESI: $464\left(\mathrm{M}+\mathrm{H}^{+}\right)$; Anal. Calcd for $\mathrm{C}_{25} \mathrm{H}_{20} \mathrm{~F}_{3} \mathrm{~N}_{5} \mathrm{O}$ : C, 64.79; $\mathrm{H}, 4.35$; N, 15.11. Found: C, 65.00; H, 4.33; N, 15.07.

(5Z)-5-((4-dimethylamino)phenylamino)methylene)-4-methyl-2-(nitrophenyl)cyclopenta-1,3-diene-1,3-dicarb onitrile (4b)

Red-orange solid (23\% yield) m.p. $280^{\circ} \mathrm{C}-282^{\circ} \mathrm{C}^{1} \mathrm{H}-\mathrm{NMR}\left(\mathrm{CDCl}_{3}\right): \delta$ (ppm) 2.48 (s, 3H, Me), 3.01 (s, $6 \mathrm{H}$, $\mathrm{NMe}_{2}$ ), 6.72 (d, 2H, J = 9.0 Hz, Ph), 7.15 (d, 2H, J = 9.0 Hz, Ph), 7.91 (d, 2H, J = 8.8 Hz, Ph), 7.97 (d, 1H, J =

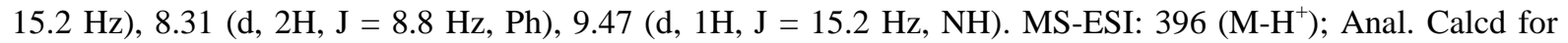
$\mathrm{C}_{23} \mathrm{H}_{19} \mathrm{~N}_{5} \mathrm{O}_{2}$ : C, 69.51; H, 4.82; N, 17.62. Found: C, 69.38; H, 4.83; N, 17.59.

(Z)-N-((2,4-dicyano-5-methyl-3-(4-nitrophenyl)-5-(2,2,2-trifluoroacetamido)cyclopenta-1,3-dien-1-yl)methyle ne)-4-(dimethylamino)aniline oxide (5b)

Blu solid (20\% yield) m.p. $169^{\circ} \mathrm{C}-171^{\circ} \mathrm{C}{ }^{1} \mathrm{H}-\mathrm{NMR}\left(\mathrm{CDCl}_{3}\right): \delta$ (ppm) 2.12 (s, 3H, Me), 3.08 (s, $6 \mathrm{H}, \mathrm{NMe}_{2}$ ), 6.65 (d, 2H, J = 9.4 Hz, Ph), 7.65 (d, 2H, J = 9.4 Hz, Ph), 7.89 (d, 2H, J = 8.6 Hz, Ph), 8.13 (s, 1H), 8.28 (s, 1H, $\mathrm{NH}$ ), 8.37 (d, $2 \mathrm{H}, \mathrm{J}=8.6 \mathrm{~Hz}, \mathrm{Ph})$. MS-ESI: $525\left(\mathrm{M}+\mathrm{H}^{+}\right)$Anal. Calcd for $\mathrm{C}_{25} \mathrm{H}_{19} \mathrm{~F}_{3} \mathrm{~N}_{6} \mathrm{O}_{4}: \mathrm{C}, 57.25 ; \mathrm{H}, 3.65 ; \mathrm{N}$, 16.02. Found: C, 57.43; H, 3.66; N, 16.05 .

$N$-(2-((E)-(4-(dimethylamino)phenylimino)methyl-3,5-dicyano-1-methyl-4-(4-nitrphenyl)cyclopenta-2,4-dieny l)-2,2,2-trifluoacetamide (6b)

Blu-violet solid (20\% yield) m.p. $116^{\circ} \mathrm{C}-118^{\circ} \mathrm{C}{ }^{1} \mathrm{H}-\mathrm{NMR}\left(\mathrm{CDCl}_{3}\right): \delta(\mathrm{ppm}) 1.93$ (s, 3H, Me), 3.08 (s, $6 \mathrm{H}$, $\mathrm{NMe}_{2}$ ), 6.70 (d, 2H, J = 9.0 Hz, Ph), 7.37 (d, 2H, J = $9.0 \mathrm{~Hz}, \mathrm{Ph}$ ), 7.94 (d, 2H, J = 8.8 Hz, Ph), 8.39 (d, 2H, J = $8.8 \mathrm{~Hz}, \mathrm{Ph}), 8.64$ (s, $1 \mathrm{H})$. MS-ESI: $509\left(\mathrm{M}+\mathrm{H}^{+}\right)$; Anal. Calcd for $\mathrm{C}_{25} \mathrm{H}_{19} \mathrm{~F}_{3} \mathrm{~N}_{6} \mathrm{O}_{3}$ : C, 59.06; $\mathrm{H}, 3.77 ; \mathrm{N}, 16.53$. Found: C, 59.25; H, 3.75; N, 16.57.

(5Z)-5-((4-(dimethylamino)phenyamino)methylene)-4-methyl-2-(2,4-dinitrophenyl)cyclopenta-1,3-diene-1,3-d icarbonitrile (4c)

Red-orange solid (28\% yield) m.p. $155^{\circ} \mathrm{C}-157^{\circ} \mathrm{C}{ }^{1} \mathrm{H}-\mathrm{NMR}\left(\mathrm{CDCl}_{3}\right): \delta(\mathrm{ppm}) 2.48$ (s, 3H, Me), 2.99 (s, $6 \mathrm{H}$, $\mathrm{NMe}_{2}$ ), 5.25 (bs, 1H, NH), 6.73 (d, 2H, J = $8.8 \mathrm{~Hz}, \mathrm{Ph}$ ), 7.19 (d, 2H, J = $8.8 \mathrm{~Hz}, \mathrm{Ph}$ ), 7.71 (d, 1H, Jo = 8.6 Hz, $\mathrm{Ph}$ ), 8.11 (d, 1H, J = $13.6 \mathrm{~Hz}$ ), 8.48 (dd, $\left.1 \mathrm{H}, \mathrm{J}_{\mathrm{o}}=8.6 \mathrm{~Hz}, \mathrm{~J}_{\mathrm{m}}=1.6 \mathrm{~Hz}, \mathrm{Ph}\right), 8.85$ (d, 1H, $\left.\mathrm{J}_{\mathrm{m}}=1.6 \mathrm{~Hz}, \mathrm{Ph}\right)$. MSESI: $441\left(\mathrm{M}-\mathrm{H}^{+}\right)$; Anal. Calcd for $\mathrm{C}_{23} \mathrm{H}_{18} \mathrm{~N}_{6} \mathrm{O}_{4}$ : C, 62.44; H, 4.10; N, 19.00. Found: C, 62.64; H, 4.08; N, 19.02.

(Z)-N-((2,4-dicyano-5-methyl-3-(2,4-dinitrophenyl)-5-(2,2,2-trifluoroacetamido)cyclopenta-1,3-dien-1-yl)met hylene)-4-(dimethyl amino)aniline oxide (5c)

Blu-violet solid (25\% yield) m.p. $177^{\circ} \mathrm{C}-179^{\circ} \mathrm{C}{ }^{1} \mathrm{H}-\mathrm{NMR}\left(\mathrm{CDCl}_{3}\right)$ : $\delta$ (ppm) 2.16 (s, 3H, Me), 3.07 (s, $6 \mathrm{H}$, $\mathrm{NMe}_{2}$ ), 6.65 (d, 2H, J = 9.2 Hz, Ph), 7.64 (d, 2H, J = $9.2 \mathrm{~Hz}, \mathrm{Ph}$ ), 7.97 (d, 1H, Jo = 8.4 Hz, Ph), 8.04 (s, 1H), 
8.63 - $8.66(\mathrm{~m}, 2 \mathrm{H}, \mathrm{NH}, \mathrm{Ph}), 9.17$ (d, $\left.1 \mathrm{H}, \mathrm{J}_{\mathrm{m}}=2.0 \mathrm{~Hz}, \mathrm{Ph}\right) .{ }^{13} \mathrm{C}-\mathrm{NMR}\left(\mathrm{CDCl}_{3}\right): \delta(\mathrm{ppm})$ 19.45, 40.24, 68.60, 111.26, 112.09, 112.18, 114.00, 116.63, 119.29, 121.14, 122.93, 124.545, 129.15, 131.05, 133.71, 136.11, 146.86, 147.17, 149.44, 152.73, 153.92, 156.70. MS-ESI: $592(\mathrm{M}+\mathrm{Na}), 568\left(\mathrm{M}-\mathrm{H}^{+}\right)$; Anal. Calcd for $\mathrm{C}_{25} \mathrm{H}_{18} \mathrm{~F}_{3}$ $\mathrm{N}_{7} \mathrm{O}_{6}$ : C, 52.73; H, 3.19; N, 17.22. Found: C, 52.90; H, 3.21; N, 17.27.

$\mathrm{N}$-(2-((E)-(4-(dimethylamino)phenylimino)methyl)-3,5-dicyano-1-methyl-4-(2,4-dinitrophenyl)cyclopenta-2,4dienyl)-2,2,2-trifluoroacetamide $(\mathbf{6 c})$

Blu solid (24\% yield) m.p. $110^{\circ} \mathrm{C}-112^{\circ} \mathrm{C}{ }^{1} \mathrm{H}-\mathrm{NMR}\left(\mathrm{CDCl}_{3}\right): \delta(\mathrm{ppm}) 1.97$ (s, 3H, Me), $3.08\left(\mathrm{~s}, 6 \mathrm{H}, \mathrm{NMe}_{2}\right)$, 6.70 (d, 2H, J = 9.0 Hz, Ph), 7.36 (d, 2H, J = 9.0 Hz, Ph), 7.96 (d, 1H, Jo = 8.0 Hz, Ph), 8.56 (s, $1 \mathrm{H}), 8.67$ (dd, $\left.1 \mathrm{H}, \mathrm{J}_{\mathrm{o}}=8.0 \mathrm{~Hz}, \mathrm{~J}_{\mathrm{m}}=2.0 \mathrm{~Hz}, \mathrm{Ph}\right), 9.20\left(\mathrm{~d}, 1 \mathrm{H}, \mathrm{J}_{\mathrm{m}}=2.0 \mathrm{~Hz}, \mathrm{Ph}\right) .{ }^{13} \mathrm{C}-\mathrm{NMR}\left(\mathrm{CDCl}_{3}\right): \delta(\mathrm{ppm})$ 23.04, 29.66, 40.25, 67.96, 111.82, 112.09, 114.07, 120.31, 121.16, 124.83, 129.07, 131.27, 133.72, 137.44, 140.13, 146.91, 149.06, 149.44, 152.13, 158.98, 166.58. MS-E SI: $576(\mathrm{M}+\mathrm{Na}), 552\left(\mathrm{M}-\mathrm{H}^{+}\right)$; Anal. Calcd for $\mathrm{C}_{25} \mathrm{H}_{18} \mathrm{~F}_{3} \mathrm{~N}_{7} \mathrm{O}_{5}$ : C, 54.25; $\mathrm{H}$, 3.28; N, 17.72. Found: C, 54.12; H, 3.29; N, 17.66 .

(5Z)-5-((4-(dimethylamino)phenylamino)methylene)2-(4-methoxyphenyl)-4-methylcyclopenta-1,3-diene-1,3-di carbonitrile (4d)

Red-orange solid (27\% yield) m.p. $215^{\circ} \mathrm{C}-218^{\circ} \mathrm{C}{ }^{1} \mathrm{H}-\mathrm{NMR}\left(\mathrm{CDCl}_{3}\right): \delta$ (ppm) 2.45 (s, 3H, Me), 2.99 (s, $6 \mathrm{H}$, $\mathrm{NMe}_{2}$ ), 3.84 (s, 3H, OMe), 6.72 (d, 2H, J = 8.8 Hz, Ph), 6.99 (d, 2H, J = 8.8 Hz, Ph), 7.12 (d, 2H, J = 8.8 Hz, Ph), 7.74 (d, 2H, J = 8.8 Hz, Ph), 7.85 (d, 1H, J = 14.8 Hz), 9.22 (d, 1H, J = 14.8 Hz, NH). MS-ESI: 383 (M+H ${ }^{+}$); Anal. Calcd for $\mathrm{C}_{24} \mathrm{H}_{22} \mathrm{~N}_{4} \mathrm{O}$ : C, 75.37; H, 5.80; N, 14.65. Found: C, 75.50; H, 5.78; N, 14.68 .

(Z)-N-((2,4-dicyano-5-methyl-3-(4-methoxyphenyl)-5-(2,2,2-trifluoroacetamido)cyclopenta-1,3-dien-1yl)meth ylene)-4-(dimethylamino)aniline oxide (5d)

Blu-violet solid (18\% yield) m.p. $201^{\circ} \mathrm{C}-203^{\circ} \mathrm{C}{ }^{1} \mathrm{H}-\mathrm{NMR}\left(\mathrm{CDCl}_{3}\right): \delta(\mathrm{ppm}) 2.09$ (s, 3H, Me), 3.06 (s, 6H, $\mathrm{NMe}_{2}$ ), 3.85 (s, 3H, OMe), 6.65 (d, 2H, J = $8.8 \mathrm{~Hz}, \mathrm{Ph}$ ), 7.01 (d, 2H, J = 8.8 Hz, Ph), 7.63 (d, 2H, J = 8.8 Hz, $\mathrm{Ph}$ ), 7.73 (d, 2H, J = 8.8 Hz, Ph), 8.01 (s, 1H), 8.23 (s, 1H, NH). MS-ESI: $510\left(\mathrm{M}+\mathrm{H}^{+}\right)$; Anal. Calcd for $\mathrm{C}_{26} \mathrm{H}_{22}$ $\mathrm{F}_{3} \mathrm{~N}_{5} \mathrm{O}_{3}$ : C, 61.29; H, 4.35; N, 13.75. Found: C, 61.45; H, 4.36; N, 13.71.

$\mathrm{N}$-(2-((E)-(4-(dimethylamino)phenylimino)methyl-3,5-dicyano-4-(4-methoxyhenyl)-1-methylcyclopenta-2,4-di enyl)-2,2,2-trifluoro acetamide (6d)

Blu-violet solid (20\% yield) m.p. $235^{\circ} \mathrm{C}-240^{\circ} \mathrm{C}{ }^{1} \mathrm{H}-\mathrm{NMR}\left(\mathrm{CDCl}_{3}\right): \delta(\mathrm{ppm}) 1.89$ (s, 3H, Me), 3.06 (s, $6 \mathrm{H}$, $\mathrm{NMe}_{2}$ ), 3.86 (s, 3H, OMe), 6.69 (d, 2H, J = 8.8 Hz, Ph), $7.01-7.05$ (m, 3H, NH, Ph), 7.35 (d, 2H, J = 9.0 Hz, $\mathrm{Ph}$ ), 7.79 (d, 2H, J = 9.0 Hz, Ph), 8.64 (s, 1H). MS-ESI: $516(\mathrm{M}+\mathrm{Na})$; Anal. Calcd for $\mathrm{C}_{26} \mathrm{H}_{22} \mathrm{~F}_{3} \mathrm{~N}_{5} \mathrm{O}_{2}$ : C, 63.28; H, 4.49; N, 14.19. Found: C, 63.47; H, 4.47; N, 14.22 .

\subsection{RX Crystallography Data}

Single crystals of $\mathbf{3 b}, \mathbf{4 a}$ and $\mathbf{5 a}$ were submitted to X-ray data collection by using a Siemens P4 four-circle or an Oxford-Diffraction Xcalibur Sapphire 3 diffractometer. The structures were solved by direct methods implemented in SHELXS-97 program [11]. The refinements were carried out by full-matrix anisotropic least- squares on $\mathrm{F}^{2}$ for all reflections for non-H atoms by means of the SHELXL-97 program [11]. Crystallographic data for the structure in this paper have been deposited with the Cambridge Crystallographic Data Centre as supplementary publication no. CCDC 1416382 (3b), CCDC 995914 (4a), CCDC 995915 (5a). Copies of the data can be obtained, free of charge, on application to CCDC, 12 Union Road, CambridgeCB2 1EZ, UK; (fax: + 44 (0) 1223 336 033; or e-mail: deposit@ccdc.cam.ac.uk).

Crystal data for $3 \mathbf{b}: \mathrm{C}_{22} \mathrm{H}_{20} \mathrm{~N}_{4} \mathrm{O}_{4}(\mathrm{CCDC} 1416382): \mathrm{M}_{\mathrm{W}}=404.42$, triclinic, space group P-1, a = 7.6387 (6) $\AA$, $\mathrm{b}=16.6184$ (12) $\AA, \mathrm{c}=17.7794$ (13) $\AA, \alpha=112.298$ (7), $\beta=94.328$ (6), $\gamma=92.519(6)^{\circ}, \mathrm{V}=2075.9$ (3) $\AA^{3}, \mathrm{Z}=$ $4, \mathrm{D}_{\mathrm{c}}=1.294 \mathrm{mg} / \mathrm{m}^{3}, \mathrm{~F}(000)=848$, crystal dimension 0.3_0.1_0.1 mm, radiation, MoK $\alpha(\lambda=0.71073 \AA), 9355$ intensity data were collected at $293(2) \mathrm{K}$, employing $\omega / 2 \theta$ scanning technique, in the range of $-10 \mathrm{~h} 9,-20 \mathrm{k} 19$, -24123; the structure was solved by a direct method, all non-hydrogen atoms were refined anisotropically from 2585 observed reflections by a full-matrix least-squares technique. $\mathrm{R}=0.0467[\mathrm{I}>2 \sigma(\mathrm{I})]$ and $\mathrm{wR}_{2}$ (all data) $=$ 0.1012

Crystal data for 4a: $\mathrm{C}_{23} \mathrm{H}_{20} \mathrm{~N}_{4}(\mathrm{CCDC} 995914): \mathrm{M}_{\mathrm{W}}=352.43$, monoclinic, space group P2 $1 / \mathrm{n}, \mathrm{a}=8.3817$ (4) $\AA$, $\mathrm{b}=12.9420$ (7) $\AA, \mathrm{c}=17.4334$ (12) $\AA, \alpha=90, \beta=98.704$ (5), $\gamma=90^{\circ}, \mathrm{V}=1869.3(1) \AA^{3}, \mathrm{Z}=4, \mathrm{D}_{\mathrm{c}}=1.252$ $\mathrm{mg} / \mathrm{m}^{3}, \mathrm{~F}(000)=744$, crystal dimension 0.4_0.3_0.1 mm, radiation, MoK $\alpha(\lambda=0.71073 \AA$ ), 4310 intensity data were collected at $293(2) \mathrm{K}$, employing $\omega / 2 \theta$ scanning technique, in the range of $-10 \mathrm{~h} 8,-16 \mathrm{k} 16,-22 \mathrm{l} 15$; the structure was solved by a direct method, all non-hydrogen atoms were refined anisotropically from 1344 observed reflections by a full-matrix least-squares technique. $\mathrm{R}=0.0426[\mathrm{I}>2 \sigma(\mathrm{I})]$ and $\mathrm{wR}_{2}($ all data $)=0.0873$ 
Crystal data for 5a: $\mathrm{C}_{25} \mathrm{H}_{20} \mathrm{~F}_{3} \mathrm{~N}_{5} \mathrm{O}_{2} \cdot \mathrm{C}_{6} \mathrm{D}_{6}(\mathrm{CCDC} 995915)$ : $\mathrm{M}_{\mathrm{W}}=479.46$, monoclinic, space group P2 $1 \mathrm{a}, \mathrm{a}=$ 11.832 (1) $\AA, \mathrm{b}=13.5134$ (9) $\AA, \mathrm{c}=14.934$ (2) $\AA, \alpha=90, \beta=95.522$ (2), $\gamma=90^{\circ}, \mathrm{V}=2376.7(4) \AA^{3}, \mathrm{Z}=4, \mathrm{D}_{\mathrm{c}}$ $=1.34 \mathrm{mg} / \mathrm{mm}^{3}, \mathrm{~F}(000)=992$, crystal dimension 0.3_0.15_0.10 mm, radiation, $\operatorname{MoK}_{\alpha}(\lambda=0.71073 \AA), 5426$ intensity data were collected at 293(2) $\mathrm{K}$, employing $\omega / 2 \theta$ scanning technique, in the range of $-13 \mathrm{~h} 15,-18 \mathrm{k} 18$, -18117; the structure was solved by a direct method, all non-hydrogen were refined anisotropically from 1703 observed reflections by a full-matrix least-squares technique. $\mathrm{R}=0.0941[\mathrm{I}>2 \sigma(\mathrm{I})]$ and $\mathrm{wR}_{2}($ all data $)=0.1829$.

\section{Result and Discussion}

Hence, when dienes 1a-d $(0.1 \mathrm{mmol})$ were left to react with $N, N$-dimethyl-4-nitrosoaniline $(0.12 \mathrm{mmol})$ in $\mathrm{CH}_{2} \mathrm{Cl}_{2}$ at room temperature for 5 - 7 days a new unexpected compound we observed. This product was also observed when the same reaction mixture was heated at $60^{\circ} \mathrm{C}$ for $7 \mathrm{~h}$. The crude reaction mixture was purified by column chromatography (ethyl acetate/petroleum ether 1/4) to give starting material and compounds 3a-d (Scheme 1).

The structure of compound $\mathbf{3 b}$ was established by X-ray analysis (Figure 2), whereas allowed MS/ESI and ${ }^{1} \mathrm{H}-\mathrm{NMR}$ and ${ }^{13} \mathrm{C}-\mathrm{NMR}$ data were sufficient to establish, by comparison, the structures of derivatives $3 \mathbf{a}$, 3c and 3d.

The reaction conditions were optimized to provide compounds 3a-d in highest yields. A solution of 1a-d (0.1 mmol) and $2(0.12 \mathrm{mmol})$ in $\mathrm{CH}_{2} \mathrm{Cl}_{2}$ was heated under $\mathrm{MW}(150 \mathrm{~W})$ for 90 minutes. In alternative, heating the same mixture in an oil bath for 7 hours at $60^{\circ} \mathrm{C}$ gave desired 3a-d in similar high yields. We have verified that increasing the temperature did not increase the yield due, which we explained considering the thermolability of the compounds 3a-d.

In order to understand the effect of the temperature on product distribution, a new set of experiments was also performed. When a mixture of dienes 1a-d $(0.1 \mathrm{mmol})$ and $N, N$-dimethyl-4-nitrosoaniline $2(0.12 \mathrm{mmol})$ neat were heating under MW irradiation $(150 \mathrm{~W})$ at $150^{\circ} \mathrm{C}$ for 15 minutes, the tlc (ethyl acetate/petroleum ether $\left.1 / 4\right)$ and ${ }^{1} \mathrm{H}-\mathrm{NMR}$ spectra of the crude reaction mixture indicated complete conversion of the starting material and appearance of compounds 4a-d, 5a-d and 6a-d (Scheme 2). The reaction products were isolated by column chromatography (ethyl acetate/petroleum ether 1/4) and further characterised via NMR spectroscopy and X-ray analysis.

RX analysis (Figure 3, Figure 4) allowed us to assign the structure of compounds 4a and 5a.

The Z configuration of compound 4a was also confirmed by NMR-NOESY experiments, which showed correlation between the 4-methyl group at $2.46 \mathrm{ppm}$ and the $\mathrm{CH}$ group at $7.89 \mathrm{ppm}$. It is to be noted that $\mathbf{4 a}$ did not rearrange to the corresponding $E$-isomer upon UV-VIS irradiation at $440 \mathrm{~nm}$.

The structure of other 4, 5 and $\mathbf{6}$ analogues was assigned by comparison of their ${ }^{1} \mathrm{H}-\mathrm{NMR}$ data (Table 1).

The analysis of the chemical shifts indicated very similar trend of the C-methyl group and the CH-N signals along the three series of compounds.

Also analysis of the MS/ESI data (molecular ion value and ms/ms fragmentation) confirms the assigned structure.

The medium basicity (due the presence of nitrosoamine) is a crucial parameter for the Ehrlich-Sachs reaction. In fact compounds 1a-d $\left(0.1 \mathrm{mmmol}\right.$ in $\left.\mathrm{CH}_{2} \mathrm{Cl}_{2}\right)$ does not react with nitrosobenzene $(0.12 \mathrm{mmmol})$ in the absence of base and under MW irradiation (solvent free) gave no conversion to corresponding compounds 4.

Conversely, in the presence of triethylamine $(0.12 \mathrm{mmmol})$ the corresponding aniline oxide was obtained from compounds $\mathbf{1 b}, \mathbf{d}\left(0.1 \mathrm{mmmol}\right.$ in $\left.\mathrm{CH}_{2} \mathrm{Cl}_{2}\right)$ and nitrosobenzene, $(0.12 \mathrm{mmmol})$, as confirmed by MS/ESI data and ${ }^{1} \mathrm{H}-\mathrm{NMR}$ spectra: the chemical shifts of the methyl group (2.15 ppm for $\mathrm{NO}_{2}$ derivative and $2.11 \mathrm{ppm}$ for OMe derivative) and those of the $\mathrm{CH}-\mathrm{N}$ proton $\left(8.18 \mathrm{ppm}\right.$ and $8.15 \mathrm{ppm}$ respectively for $\mathrm{NO}_{2}$ and $\mathrm{OMe}_{\mathrm{Me}}$ rivatives) are diagnostic for the assignement of the structure. In addition compounds 5 (and $\mathbf{6}$ in trace) were obtained from $1\left(0.1 \mathrm{mmmol}\right.$ in $\left.\mathrm{CH}_{2} \mathrm{Cl}_{2}\right)$ and $2(0.12 \mathrm{mmmol})$ and trimethylamine $(0.12 \mathrm{mmmol})$ without heating.

Fulvenes requires higher temperatures: 4a-d together with 5 a-d were also obtained by heating of $\mathbf{1 a - d}(0.1$ $\mathrm{mmol})$ and $2(0.12 \mathrm{mmol})$ at $100^{\circ} \mathrm{C}$ in oil bath, solvent free, for 3 hours.

The formation of 3a-d may be accounted by the following mechanisms (Scheme 3).

We envisaged that, following an initial hetero Diels-Alder reaction [2], deprotonation of $\mathrm{NHCOCF}_{3}$ group lead to formation of an open intermediate which rearranged to a dihydrofuran. Finally, the hydrolysis (the reaction is made in non-anhydrous conditions) of the trifluoroacetamidate group gave the observed compounds 3a-d. 


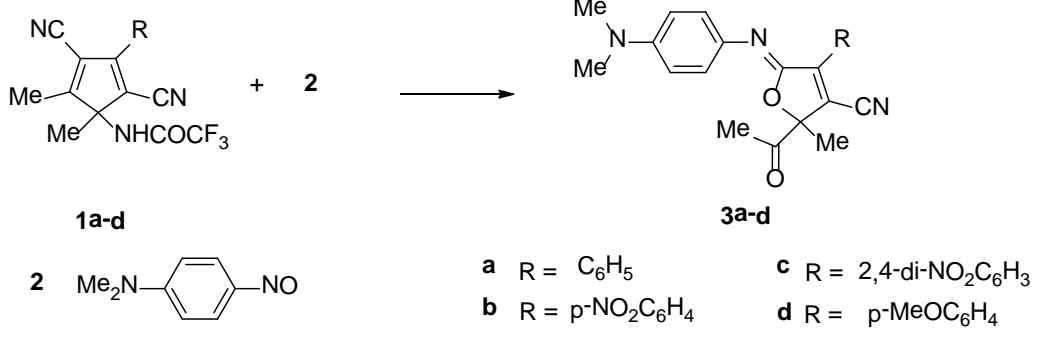

Scheme 1. 1a-d (0.1 mmol) and $2(0.12 \mathrm{mmol})$ in $\mathrm{CH}_{2} \mathrm{Cl}_{2}(3 \mathrm{ml})$ at RT for 5 - 7 days or $\mathrm{T}=60^{\circ} \mathrm{C}$ in bath oil for $7 \mathrm{~h}$, or heating in MW $150 \mathrm{~W}$ for 90 minutes.

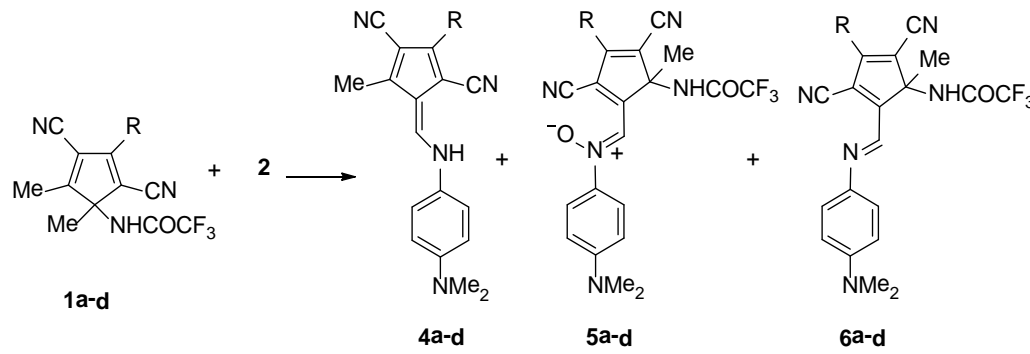
a $\mathrm{R}=\mathrm{C}_{6} \mathrm{H}_{5}$
c $\mathrm{R}=2,4-\mathrm{di}-\mathrm{NO}_{2} \mathrm{C}_{6} \mathrm{H}_{3}$
b $\mathrm{R}=\mathrm{p}-\mathrm{NO}_{2} \mathrm{C}_{6} \mathrm{H}_{4}$
d $\mathrm{R}=\mathrm{p}-\mathrm{MeOC}_{6} \mathrm{H}_{4}$
$2 \mathrm{Me}_{2} \mathrm{~N} \longrightarrow \mathrm{NO}$

Scheme 2. 1a-d $(0.1 \mathrm{mmol})$ and $2(0.12 \mathrm{mmol})$ in $\mathrm{MW}$ at $\mathrm{T}=150^{\circ} \mathrm{C}, 150 \mathrm{~W}$ for $15^{\prime}$ solvent free or heating in oil bath at $\mathrm{T}=100^{\circ} \mathrm{C}$ for $3 \mathrm{~h}$ solvent free.

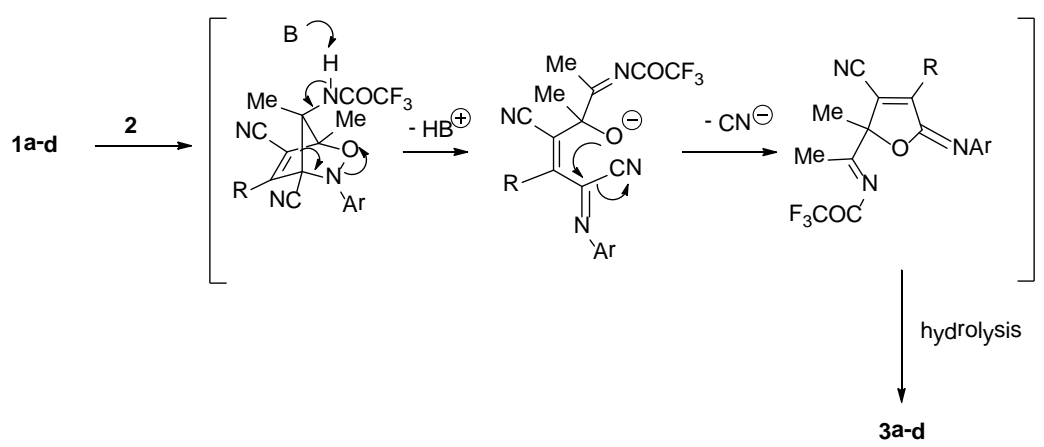

Scheme 3. Proposed mechanism for the formation of compounds 3a-d.

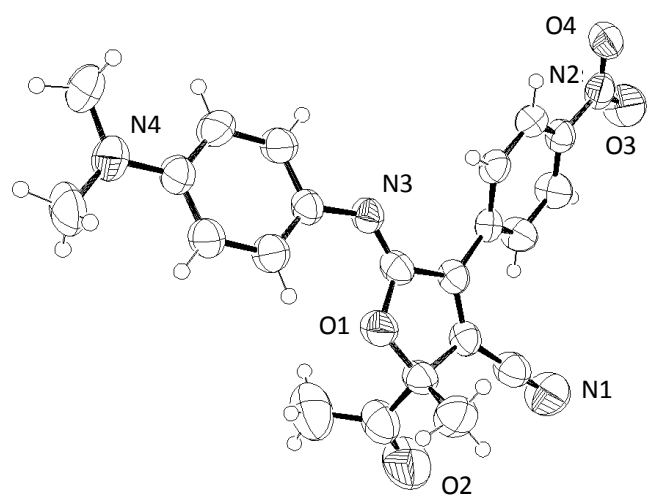

Figure 2. Crystal structure of compound 3b (CCDC1416382). Ellipsoids enclose 50\% probability. 


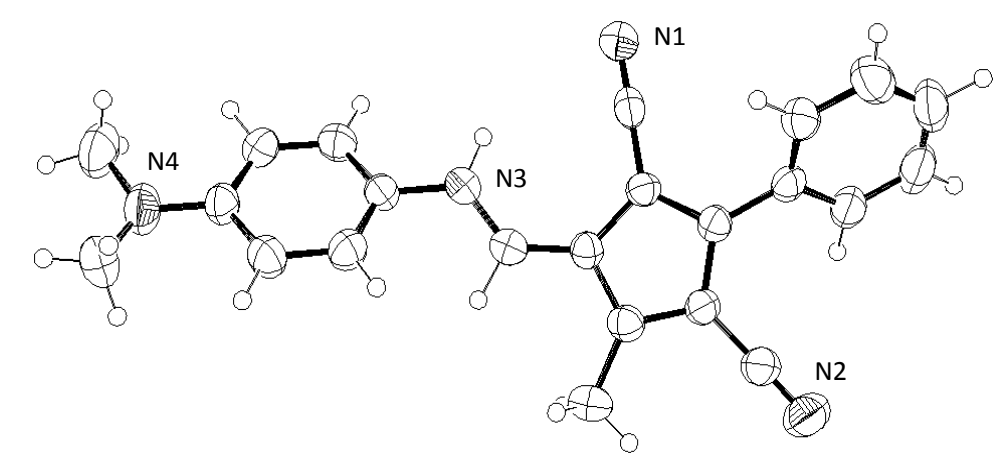

Figure 3. Crystal structure of compound 4a (CCDC995914). Ellipsoids enclose $50 \%$ probability.

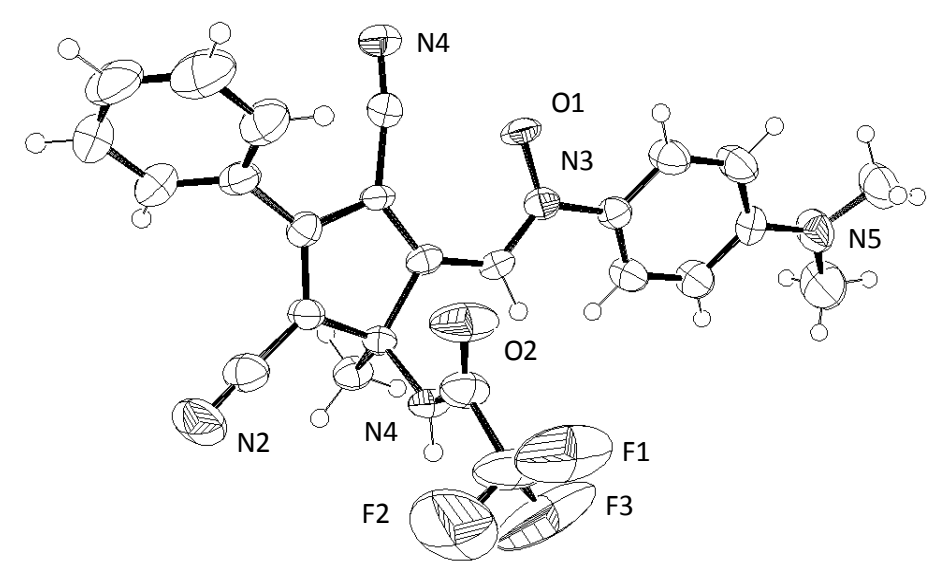

Figure 4. Crystal structure of compound 5a (CCDC995915). Ellipsoids enclose $50 \%$ probability.

Table 1. Selected proton NMR of compounds $\mathbf{4 a - d , 5 a - d ~ a n d ~} \mathbf{6 a - d .}$

\begin{tabular}{|c|c|c|c|c|c|c|}
\hline \multirow[t]{2}{*}{$\mathrm{R}$} & \multicolumn{2}{|c|}{ 4a-d } & \multicolumn{2}{|c|}{ 5a-d } & \multicolumn{2}{|c|}{$6 a-d$} \\
\hline & $\mathrm{Me}$ & $\mathrm{CH}-\mathrm{N}$ & $\mathrm{Me}$ & $\mathrm{CH}-\mathrm{N}$ & $\mathrm{Me}$ & CH-N \\
\hline Phenyl & 7.89 & 2.46 & 2.11 & 8.11 & 1.91 & 8.65 \\
\hline 4- $\mathrm{NO}_{2}$ Phenyl & 7.97 & 2.48 & 2.12 & 8.13 & 1.93 & 8.64 \\
\hline 2,4diNO ${ }_{2}$ Phenyl & 8.11 & 2.48 & 2.16 & 8.04 & 1.97 & 8.56 \\
\hline 4-OMePhenyl & 7.85 & 2.45 & 2.09 & 8.01 & 1.89 & 8.64 \\
\hline
\end{tabular}

The formation of the imines 6a-d and nitrones 5a-d could be explained by an Ehrlich-Sachs condensation mechanism involving nitroso group and acidic methyl group, followed by partial oxidation to 5 by the excess of reagent [3].

The formation of fulvenes can be hypothesized as deriving from 6a-d considering that nitrosobenzene, in presence of carbanions with good leaving groups gave N-oxides [3]. In this case, the acidic NH was deprotonated to an $\mathrm{N}$-anion able to carry a nucleophilic attack to the electrophilic nitrosoaniline. In this context, the fulvene behaved as a leaving group generating compounds 4a-d and trifluoroacetyldiazene-oxide 7 (Scheme 4).

We have carried out some mechanistic studies that support this interpretation. For example, heating compounds 6a-d in the presence of $N, N$-dimethyl-4-nitrosoaniline 2, under neat conditions, provided fulvenes which were characterised by t.l.c. mass spectrometry and ${ }^{1} \mathrm{H}-\mathrm{NMR}$. In addition, the presence of trifluoroacetyl- 


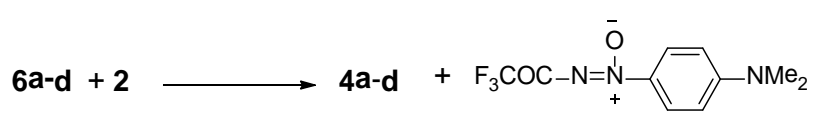

7

Scheme 4. Proposed reaction for the formation of compounds 4a-d.

diazene-oxide 7 could be evidenced in the crude mixture [12].

\section{Conclusion}

In conclusion, we have shown that the reaction of compounds $\mathbf{1}$ and $\mathbf{2}$ could be in two diverse directions providing either substituted dihydrofuran or fulvenes depending upon conditions applied. The increase in temperature does not lead to the cycloaddition reaction, but a different mechanism occurs. The interest on synthetic methodologies leading to 2,5-dihydrofuran and imino-2,5-dihydrofuran derivatives [13] is very considerable. These structures are subunit of several bioactive natural and synthetic products [14] [15]. Fulvenes have high relevance as synthons [16] [17] and materials [18]-[24]. This report will provide a fast and easy protocol to execute the preparation of the abovementioned heterocycles cores.

\section{Acknowledgements}

The work was financially supported by the funds of University of Siena. We would like to thank Dr. Sara Draghi for ESI-MS analysis, Dr. Iacopo Barbetti for laboratory experimental assistance and the Centro di Analisi e Determinazioni Strutturali of the University of Siena for X-ray data collection.

\section{References}

[1] Fusi, S., Ponticelli, F., Ventura, A. and Adamo, F.A. (2008) Rearrangement of 3,5-Dicyano-1,4-dihydropyridines to Densely Functionalized Cyclopentadienes. Tetrahedron Letters, 49, 5820-5822. http://dx.doi.org/10.1016/j.tetlet.2008.07.129

[2] Fusi, S., Ferrini, S. and Ponticelli, F. (2011) [4+2] Cycloaddition on Densely Functionalized Cyclopentadiene. Tetrahedron Letters, 52, 6911-6915. http://dx.doi.org/10.1016/j.tetlet.2011.10.058

[3] Zuman, P. and Shah, B. (1994) Addition, Reduction, and Oxidation Reactions of Nitrosobenzene. Chemical Review, 94, 1621-1641. http://dx.doi.org/10.1021/cr00030a007

[4] Vogt, P.F. and Miller, M.J. (1998) Development and Applications of Amino Acid-Derived Chiral Acylnitroso Hetero Diels-Alder Reactions. Tetrahedron, 54, 1317-1348. http://dx.doi.org/10.1016/S0040-4020(97)10072-2

[5] Da Silva Machado, F.L. (2008) Nitrosobenzene. Synlett, 19, 3075-3076. http://dx.doi.org/10.1055/s-2008-1067280

[6] Lemire, A., Beaudoin, D., Grenon, M. and Charetten, A.B. (2005) [4+2] Cycloaddition of 2-Substituted 1,2Dihydropyridines with Nitrosobenzene: Asymmetric Synthesis of Trans-2-substituted 3-Amino-1,2,3,6-tetrahydropyridines. The Journal of Organic Chemistry, 70, 2368-2371. http://dx.doi.org/10.1021/jo048216x

[7] Jones, A.L. and Snyder, J.K. (2010) Synthesis of Unique Scaffolds via Diels-Alder Cycloadditions of Tetrasubstituted Cyclohexadienes. Organic Letters, 12, 1592-1595. http://dx.doi.org/10.1021/ol100318f

[8] Yamamoto, Y., Momiyama, N. and Yamamoto, H. (2004) Enantioselective Tandem O-Nitroso Aldol/Michael Reaction. Journal of American Chemical Society, 126, 5962-5963. http://dx.doi.org/10.1021/ja049741g

[9] Zhong, G.F. and Yu, Y.P. (2004) Enantioselective Synthesis of Allylic Alcohols by the Sequential AminoxylationOlefination Reactions of Aldehydes under Ambient Conditions. Organic Letters, 6, 1637-1639. http://dx.doi.org/10.1021/ol049524k

[10] Zimmer, R., Buchholz, M., Collas, M., Angermann, J., Homann, K. and Reissig, H.-U. (2010) 1,2-Oxazines as Building Blocks for Stereoselective Synthesis: Preparation of Oxygen-Substituted 1,2-Oxazines, Either by Alcohol Addition or by Epoxidation, and Subsequent Hydrogenation Leading to 1,2-Amino Alcohols and Pyrrolidines. European Journal of Organic Chemistry, 2010, 4111-4121. http://dx.doi.org/10.1002/ejoc.201000425

[11] Sheldrick, G.M. (1997) SHELXS-97, rel.97-2: A Program for Automatic Solution of Crystal Structure. University of Göttingen, Göttingen.

[12] Churakov, A.M., Ioffe, S.L. and Tartakovskii, V.A. (1996) Synthesis of l-Aryl-2-nitrodiazene 1- $N$-oxides. Mendeleev Communication, 6, 20-22. http://dx.doi.org/10.1070/MC1996v006n01ABEH000560 
[13] Avetysan, A.A. and Karapetyan, L.V. (2013) Synthesis and Chemical Transformations of Bis-2-imino-2,5-dihydrofurans. Chemistry of Heterocyclic Compounds, 48, 1613-1620. http://dx.doi.org/10.1007/s10593-013-1182-8

[14] Awaad, A.S., Nabilah AJ.A. and Zain, M.E. (2012) New Antifungal Compounds from Aspergillus terreus Isolated from Desert Soil. Phytotherapy Research, 26, 1872-1877. http://dx.doi.org/10.1002/ptr.4668

[15] Mackman, R.L., Ray, A.S., Hui, H.C., Zang, L., Birkus, G., Boojamra, C.G., Desai, M.C., Douglas, J.L., Gao, Y., Grant, D., Laflamme, G., Lin, K.Y., Markevitch, D.Y., Mishra, R., McDermott, M., Pakdaman, R., Petrkovsky, O.V., Vela, J.E. and Cihlar, T. (2010) Discovery of GS-9131: Design, Synthesis and Optimization of Amidate Prodrugs of the Novel Nucleoside Phosphonate HIV Reverse Transcriptase (RT) Inhibitor GS-9148. Biorganic and Medicinal Chemistry, 18, 3606-3617. http://dx.doi.org/10.1016/j.bmc.2010.03.041

[16] Coskun, N. and Erden, I. (2011) An Efficient Catalytic Method for Fulvene Synthesis. Tetrahedron, 67, 8607-8614. http://dx.doi.org/10.1016/j.tet.2011.09.036

[17] Enk, B., Kopacka, H., Wurst, K., Muller, T. and Bildstein, B. (2009) An Efficient, Modular Route to New 2-Acyl-6aminopentafulvenes and Planar-Chiral [N,O]-Functionalized Pentamethylruthenocenes. Organometallics, 28, 55755586. http://dx.doi.org/10.1021/om900549c

[18] Kedziorek, M., Mayer, P. and Mayr, H. (2009) Nucleophilic Reactivities of Azulene and Fulvenes. European Journal of Organic Chemistry, 2009, 1202-1206. http://dx.doi.org/10.1002/ejoc.200801099

[19] Muthusamy, S., Babu, S.A., Gunanathan, C., Suresh, E. and Dastidar, P. (2001) Novel Intermolecular [3+2] Cycloaddition Reaction of Carbonyl Ylides with Fulvenes: Entry into the Oxatetracyclo [6.5.1.01,6.09,13] Tetradecene Ring System. Synlett, 2001, 1407-1410.

[20] Hong, B.C., Shr, Y.J., Wu, J.L., Gupta, A.K. and Lin, K.J. (2002) Novel [6+2] Cycloaddition of Fulvenes with Alkenes: A Facile Synthesis of the Anislactone and Hirsutane Framework. Organic Letters, 4, 2249-2252. http://dx.doi.org/10.1021/ol026103z

[21] Lee, K.J., Choi, J.K., Yum, E.K. and Cho, S.Y. (2009) Novel 1,3-Dipolar Cycloadditions of Fulvenes and Hydrazonyl Chlorides: A Facile Synthesis of the Cyclopenta[d]pyridazines. Tetrahedron Letters, 50, 6698-6700. http://dx.doi.org/10.1016/j.tetlet.2009.09.086

[22] Poirier, M.A. (1992) US 5113325 A 19920602.

[23] Arbiser, J.L. (2008) US 20080275016 A1 20081106.

[24] Hogan, M., Cotter, J., Claffey, J., Gleeson, B., Wallis, D., O’Shea, D.L. and Tacke, M. (2008) Synthesis and Cytotoxicity Studies of New (Dimethylamino)-Functionalised and 7-Azaindole-Substituted “Titanocene” Anticancer Agents (7-Azaindole = 1H-Pyrrolo[2,3-b]pyridine). Helvetica Chimica Acta, 91, 1787-1797. http://dx.doi.org/10.1002/hlca.200890191

\section{Submit or recommend next manuscript to SCIRP and we will provide best service for you:}

Accepting pre-submission inquiries through Email, Facebook, Linkedin, Twitter, etc A wide selection of journals (inclusive of 9 subjects, more than 200 journals)

Providing a 24-hour high-quality service

User-friendly online submission system

Fair and swift peer-review system

Efficient typesetting and proofreading procedure

Display of the result of downloads and visits, as well as the number of cited articles

Maximum dissemination of your research work

Submit your manuscript at: http://papersubmission.scirp.org/ 